\title{
Type I Monteggia lesion and associated fracture of the distal radius and ulna metaphysis in a child
}

\author{
Noel Peter, BMedSci (Hons) BMBS; ${ }^{*}$ Sein Myint, MBBS, $\mathrm{MSc}^{\dagger}$
}

\begin{abstract}
We report a rare case of a type I Monteggia lesion with an associated fracture of the distal radius and ulna metaphysis in a child. We discuss the mechanism of injury and the importance of thorough clinical examination in childhood injuries.

Key words: Monteggia, forearm injury, child, Monteggia combination injuries, metaphyseal injuries, Bado classification, radio-capitellar line, radius, ulna, epiphysis, treatment outcome

\section{RÉSUMÉ}

Nous signalons un cas rare de lésion de Monteggia de type I avec fracture associée du radius distal et de la métaphyse du cubitus chez un enfant. Nous discutons du mécanisme du traumatisme et de l'importance d'un examen clinique approfondi des blessures chez les enfants.
\end{abstract}

\section{Introduction}

The Monteggia lesion is a term used to describe a fracture of the ulna in association with a dislocation of the radiocapitellar joint and was first described by Giovanni Batista Monteggia of Milan in 1814. He observed the original 2 injuries in adult human cadavers and described it as a "traumatic lesion distinguished by a fracture of the proximal third of the ulna and an anterior dislocation of the proximal epiphysis of the radius."

It was not until 1967 that Bado renamed the term "Monteggia lesion" and classified the adult injury into 4 types, depending on the direction of the radial head dislocation and angulation of the fracture in the ulna ${ }^{2}$ (Box 1). Even though Monteggia fractures were initially described in adults, the peak incidence occurs between the ages of 4 and 10 years. Even in children it is rare, representing less than $0.4 \%$ of forearm fractures in children. Based on the current classification, a Bado type I injury is the most common (59\%), followed by the type III injury (26\%). ${ }^{3,4}$

\section{Mechanism}

The mechanism of injury of a type I lesion has been studied extensively, however, there is still much debate regarding its true etiology. One of the most widely accepted theories was postulated by Evans ${ }^{1}$ who, by subjecting the forearms of adult cadavers to different forces, concluded that the lesion is a result of a hyperpronation injury. Other theories that have been suggested include the "direct blow theory" by Speed $^{5}$ and the "hyperextension theory" by Tompkins.

Box 1. The Bado ${ }^{2}$ classification of Monteggia fracturedislocations

- Type I: Fracture of the proximal or middle third of the ulna with anterior dislocation of the radial head

- Type II: Fracture of the proximal or middle third of the ulna with posterior dislocation of the radial head

- Type III: Fracture of the ulnar metaphysis with lateral dislocation of the radial head

From the *Orthopedic Department and the †Accident and Emergency Department, Royal Berkshire Hospital, Reading, UK

Received: Jan. 17, 2007; revisions received: Mar. 21, 2007; accepted: Mar. 21, 2007

This article has been peer reviewed.

Can J Emerg Med 2007;9(5):383-6 
This paper reports a case of an unusual combination of a type I Monteggia lesion with an associated ipsilateral distal radial-ulna metaphyseal fracture in a child. We hope this report emphasizes the need for meticulous clinical examination in injuries involving children.

\section{Case report}

A 5-year-old boy fell off a climbing-obstacle frame at a playground at a height of 2-3 $\mathrm{m}$ onto his right, dominant hand and was brought to the emergency department complaining of a painful right wrist. He denied any symptoms of pain in his right elbow, although he was reluctant to move it. Clinical assessment demonstrated a tender, swollen right wrist with reduced range of motion in the right wrist and elbow joint. There was no neurovascular compromise. Initial radiographs revealed that the child had sustained a closed right distal radial and ulna metaphyseal fracture (Fig. 1). Additional imaging of the right elbow revealed an associated anterior dislocation of the head of the radius, and a fracture of the ulna diaphysis with anterior angulation. The injury was synonymous with a closed right type I Monteggia lesion (Fig. 2).

A closed reduction under general anesthesia was undertaken later that day. Reduction of the radial head, and the other injuries were confirmed by imaging in the operating room. The arm was immobilized in an above elbow plasterof-Paris cast. The patient had an overnight stay on the ward for general limb observations and was discharged home the following morning.

He was followed-up in a fracture clinic weekly for the next 4 weeks. Serial radiographs demonstrated good bony union, with no obvious loss of alignment (Fig. 3). At week 6 , the patient's range of elbow movement was $10^{\circ}-110^{\circ}$, with full range of motion at his wrist. He had no discomfort and was back to full activities. At 12 weeks post injury, he demonstrated no limitation of motion in the affected joints. His only symptom, noticed by his teachers in school, was that his handwriting appeared different when he attempted long sentences. Both parents and teachers felt that this change was new, and had only been noticed post injury. The patient demonstrated no other limitations of activities or function.

\section{Discussion}

Monteggia lesions are uncommon in childhood, accounting for only $0.4 \%$ of forearm fractures. In childhood cases, Monteggia lesions often are found to be the result of lowenergy trauma leading to a closed, non-comminuted frac- ture in an otherwise uninjured child. ${ }^{3,4}$ In adult cases, such injury typically results from high energy trauma, which may clearly lead to open and comminuted fractures.

Although Bado expanded the classification of the injury into 4 types, recent studies have shown that this reclassification carries no significant prognostic value. ${ }^{3}$ It has been useful in the planning of closed reduction in children, but does not predict long-term outcome. Some investigators (such as Letts and colleagues, and Wiley and Galey) have suggested different systems for classification of this injury in children. ${ }^{4,7}$

Unfortunately, the diagnosis of this injury is frequently missed. Gleeson and Beattie reported that the diagnosis of Monteggia fracture-dislocations in children was missed in $50 \%$ of cases by senior house officers in accident and emergency departments, and by $25 \%$ of senior radiolo-

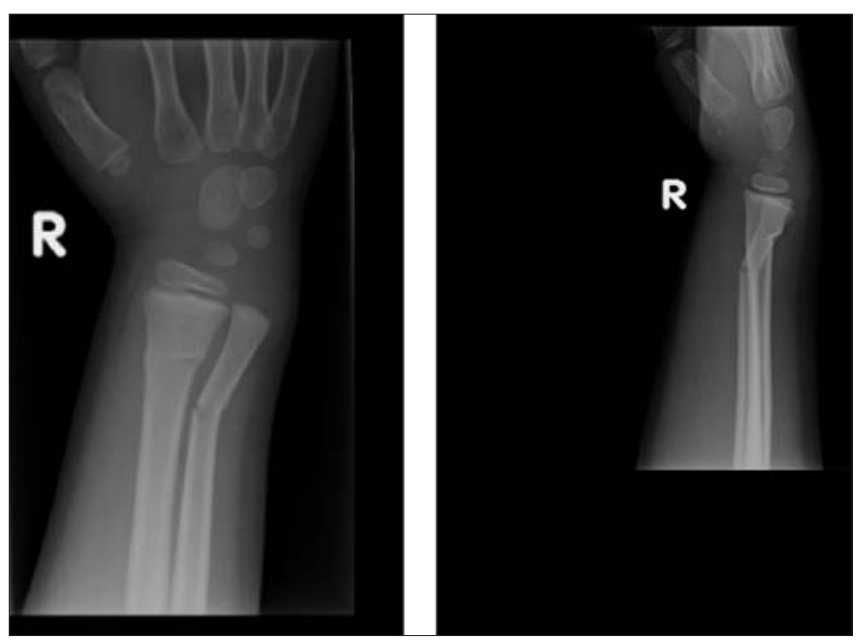

Fig. 1. Initial radiograph with distal radius and ulna metaphyseal fracture.

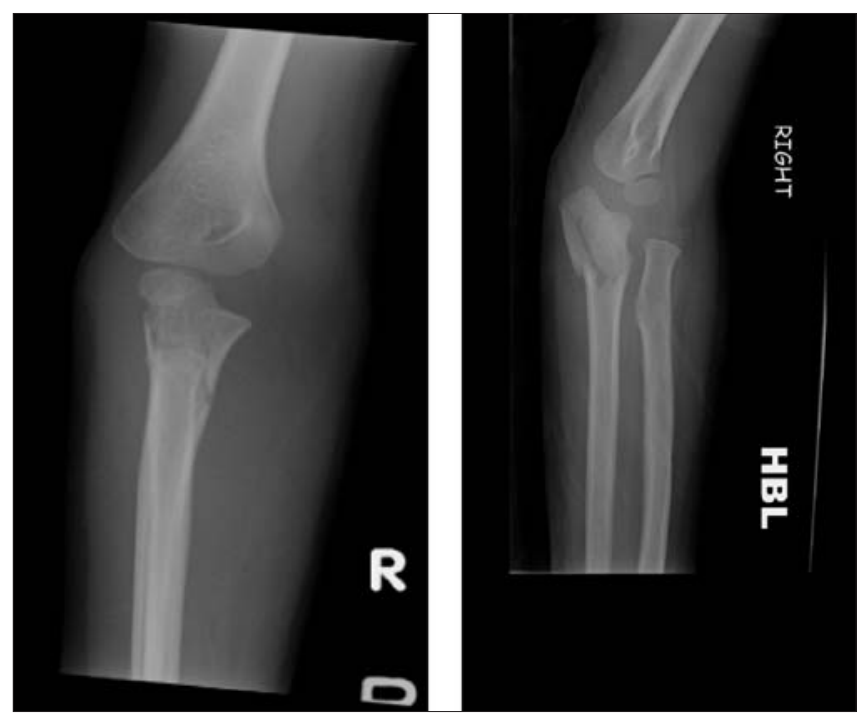

Fig. 2. Initial radiograph depicting ipsilateral closed right Monteggia lesion. 
gists. " "Missed" Monteggia lesions are associated with significant limb dysfunction and are difficult to treat. Properly assessing the nature of this injury in a timely fashion is imperative to preventing permanent disability. Complications arising from "missed" Monteggia lesions include recurrent subluxation of the radial head and associated nerve palsies.

Closed reductions of forearm fractures in children are pre-

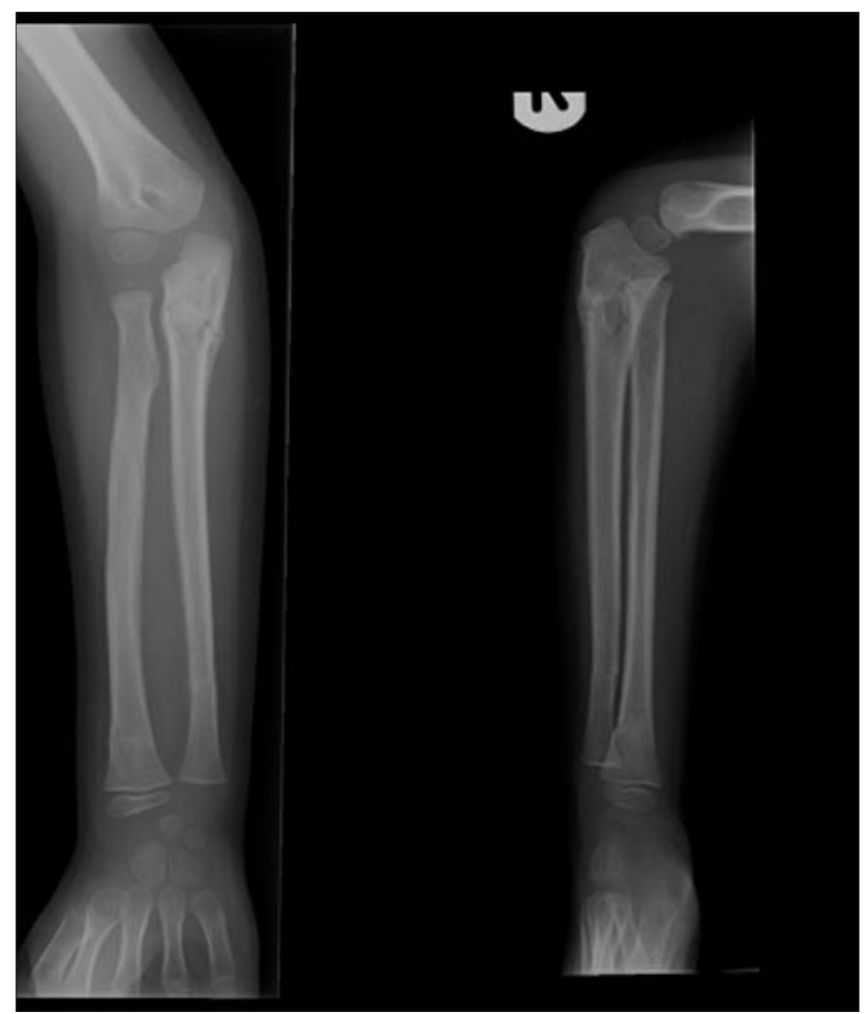

Fig. 3. Four weeks post reduction. Monteggia lesion with associated distal radial and ulna metaphyseal fracture.

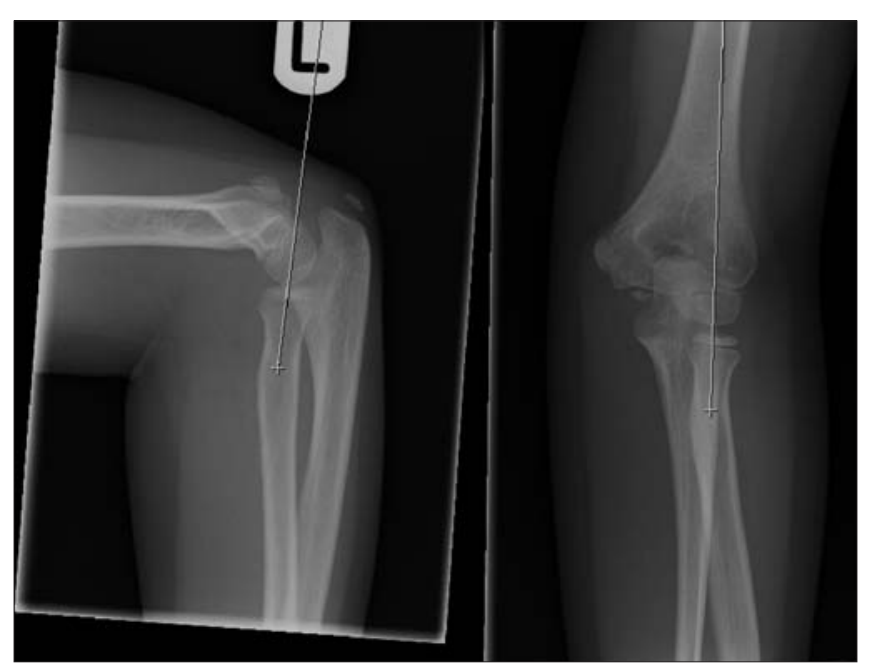

Fig. 4. A lateral and anterior-posterior radiograph of a child's elbow. The radiocapitellar line is superimposed on these radiographs. ferred because of the capacity to remodel; as long as the physes are open, remodelling can occur. However, rotational deformity does not typically correct with remodelling.

If closed reduction fails, further operative intervention should follow without delay. In terms of the management of "missed" Monteggia lesions, the treatment options are far more complex and controversial. It varies from watchful waiting to operative measures, such as open reduction and stabilization of the radial head, annular ligament repair or reconstruction, or an osteotomy of the ulna and radius (or both). ${ }^{9}$ Essentially, internal fixation of these injuries in children should be reserved for cases where closed reduction is unsuccessful. ${ }^{10-12}$

Previous case reports have described Monteggia lesions with associated ipsilateral epiphyseal injuries. ${ }^{13}$ However, a simultaneous Monteggia type I lesion with an ipsilateral distal radius ulna metaphyseal injury is an unusual combination and has rarely been reported in the literature. The exact mechanism through which the injury was sustained in this child is unclear. It is conceivable that the child fell on the outstretched pronated hand with such a great impact that it resulted in the fracture of the distal radius and ulna. Subsequently, rotational forces generated from the trunk could have resulted in the Monteggia lesion.

\section{Clinical assessment}

The clinical assessment of an injured child in the acute stage may be limited by the amount of pain, an unclear description of symptoms and high anxiety levels from the patient and the parents. Monteggia lesions are easily missed in the presence of other obvious injuries and are associated with high morbidity and complications if treatment is delayed.

Disruption of the radiocapitellar line is a useful radiological sign in assessing suspected elbow injuries in children. ${ }^{4}$ It is best applied to a true lateral projection of the joint, by drawing a line through the centre of the radial head and neck (Fig. 4). The line should pass through the centre of the capitellum. This alignment should remain intact regardless of the degree of flexion or extension of the elbow.

\section{Conclusion}

Whenever a fracture of a long bone is noted, the joints above and below should be evaluated using radiographs in orthogonal planes. If one of the forearm bones is injured, look for injury in the other bone and associated joints of the forearm, elbow and wrist. A clinician should carry out a thorough clinical examination, always looking for associated injuries when treating children involved in trauma. 
Competing interests: None declared.

\section{References}

1. Evans EM. Pronation injuries of the forearm with special reference to the anterior Monteggia fracture. J Bone Joint Surg Br 1949;31B:578-88.

2. Bado JL. The Monteggia lesion. Clin Orthop Relat Res 1967; 50:71-86.

3. Givon U, Pritisch M, Levy O, et al. Monteggia and equivalent lesions. Clin Orthop Relat Res 1997;337:208-15.

4. Letts M, Locht R, Wiens J. Monteggia fracture-dislocation in children. J Bone Joint Surg Br 1985;67B:724-7.

5. Speed JS. Treatment of fractures of the ulna with dislocation of the head of the radius. JAMA 1940;115:1699.

6. Tompkins DG. The anterior Monteggia fracture; observations of etiology and treatment. J Bone Joint Surg Am 1971;53:1109-14.
7. Wiley JJ, Galyet JP. Monteggia injuries in children. J Bone Joint Surg Br 1985;67B:728-31.

8. Gleeson AP, Beattie TF. Monteggia fracture-disclocation in children. J Accid Emerg Med 1994;11:192-4.

9. Rodgers WB, Waters PM, Hall JE. Chronic Monteggia lesions in children. J Bone Joint Surg Am 1996;78A:1322-9.

10. Peiro A, Andres F, Fernandez-Esteve F. Acute Monteggia lesions in children. J Bone Joint Surg Am 1977;59A:92.

11. Reckling FW. Unstable fracture-dislocations of the forearm. J.Bone Joint Surg Am 1982;64A:857.

12. Smith F. M. Monteggia fracture. Surg Gynecol Obstet 1947;85:630.

13. Kristiansen B, Eriksen AF. Simultaneous type II Monteggia lesion and fracture-separation of the lower radial epiphysis. Injury $1986 ; 17: 51-2$.

Correspondence to: Dr. Noel Peter, 24 Amber Close, Earley, Reading UK RG6 7ED; mzytnap@yahoo.com 